\title{
Strengthened Information-theoretic Bounds on the Generalization Error
}

\author{
Ibrahim Issa, Amedeo Roberto Esposito, Michael Gastpar \\ EPFL \\ \{ibrahim.issa,amedeo.esposito,michael.gastpar\}@epfl.ch,
}

\begin{abstract}
The following problem is considered: given a joint distribution $P_{X Y}$ and an event $E$, bound $P_{X Y}(E)$ in terms of $P_{X} P_{Y}(E)$ (where $P_{X} P_{Y}$ is the product of the marginals of $P_{X Y}$ ) and a measure of dependence of $X$ and $Y$. Such bounds have direct applications in the analysis of the generalization error of learning algorithms, where $E$ represents a large error event and the measure of dependence controls the degree of overfitting. Herein, bounds are demonstrated using several information-theoretic metrics, in particular: mutual information, lautum information, maximal leakage, and $J_{\infty}$. The mutual information bound can outperform comparable bounds in the literature by an arbitrarily large factor.
\end{abstract}

\section{INTRODUCTION}

One of the main challenges in designing learning algorithms is guaranteeing that they generalize well [1] [4]. The analysis is made especially hard by the fact that, in order to handle large data sets, learning algorithms are typically adaptive. A recent line of work initiated by Dwork et al. [5]-[7] shows that differentially private algorithms provide generalization guarantees. More recently, Russo and Zou [8], and Xu and Raginsky [9], provided an information-theoretic framework for this problem, and showed that the mutual information (between the input and output of the learning algorithm) can be used to bound the generalization error, under a certain assumption. Jiao et al. [10] and Issa and Gastpar [11] relaxed this assumption and provided new bounds using new information-theoretic measures.

The aforementioned papers mainly study the expected generalization error. In this paper, we focus instead the probability of an undesirable event (e.g., large generalization error in the learning setting). In particular, given an event $E$ and a joint distribution $P_{X Y}$, we bound $P_{X Y}(E)$ in terms of $P_{X} P_{Y}(E)$ (where $P_{X} P_{Y}$ is the product of the marginals of $P_{X Y}$ ) and a measure of dependence between $X$ and $Y$.

A bound of this form has been previously derived [12]-[14] where the measure of dependence is mutual information, $I(X ; Y)$. We present a new bound in terms of mutual information, which can outperform the existing one by an arbitrarily large factor. Moreover, we prove a new bound using lautum information (a measure introduced by Palomar and Verdú [15]). We demonstrate two further bounds using maximal leakage $[16,17]$ and $J_{\infty}(X ; Y)$ (which was recently introduced by Issa and Gastpar [11]). One advantage of the latter two bounds is that they have a closed-form expression and depend on $P_{X Y}$ only through $P_{Y \mid X}$, hence they are more amenable to analysis.

\section{KL DIVERGENCE BOUNDS}

Let $P_{X Y}$ be a joint probability distribution on alphabets $\mathcal{X} \times \mathcal{Y}$, and let $E \subseteq \mathcal{X} \times \mathcal{Y}$ be some ("undesirable") event. We want to bound $P_{X Y}(E)$ in terms of $P_{X} P_{Y}(E)$ (where $P_{X} P_{Y}$ is the product of the marginals induced by the joint $P_{X Y}$ ) and a measure of dependence between $X$ and $Y$.

\section{A. Mutual Information Bounds}

To this end, consider the following intermediate problem: let $P$ and $Q$ be two probability distributions on an alphabet $\mathcal{Z}$, and let $E \subseteq \mathcal{Z}$ be some event. We will bound $P(E)$ in terms of $Q(E)$ and $D(P \| Q)$. Then by replacing $P$ by $P_{X Y}$ and $Q$ by $P_{X} P_{Y}$, we get a bound for our desired setup in terms of the mutual information $I(X ; Y)=D\left(P_{X Y} \| P_{X} P_{Y}\right)$.

Theorem 1: Given $q \in(0,1)$, define $f_{q}:[q, 1] \rightarrow$ $\mathbb{R}_{+}$as $f_{q}(p)=D(p \| q)$. Then, $f_{q}(p)$ is a strictly increasing function of $p$. Given any event $E$ and pair of distributions $P$ and $Q$ with $D(P \| Q) \leq \log \frac{1}{Q(E)}$,

$$
P(E) \leq f_{Q(E)}^{-1}(D(P \| Q)) .
$$

In particular, given an event $E \subseteq \mathcal{X} \times \mathcal{Y}$ and a joint distribution $P_{X Y}$ satisfying $I(X ; Y) \leq \log \frac{1}{P_{X} P_{Y}(E)}$,

$$
P_{X Y}(E) \leq f_{P_{X} P_{Y}(E)}^{-1}(I(X ; Y)) \text {. }
$$

Proof: Note that $\frac{d f_{q}(p)}{d p}=\log \left(\frac{p}{q} \frac{1-q}{1-p}\right)>0$ for $p>$ $q$, hence $f_{q}(p)$ is strictly increasing. Moreover, the range of $f_{q}(p)$ is $[0, \log (1 / q)]$, so (1) is well defined.

If $P(E) \leq Q(E)$, then (1) holds trivially since $f_{Q(E)}^{-1}(D(P \| Q)) \geq Q(E)$ by the definition of $f$. 
Otherwise, if $P(E)>Q(E)$, then $f_{Q(E)}(P(E))=$ $D(P(E) \| Q(E)) \leq D(P \| Q)$, where the second inequality follows from the data processing inequality. Since $f_{q}$ is strictly increasing, then so is $f_{q}^{-1}$. Hence $P(E) \leq f_{Q(E)}^{-1}(D(P \| Q))$.

Remark 1: The bound above is tight in the following sense. Let $g:[0,1] \times \mathbb{R}_{+} \rightarrow[0,1]$ be such that, given any alphabet $\mathcal{Z}$ and event $E \subseteq \mathcal{Z}$, and any two distributions $P$ and $Q$ on $\mathcal{Z}, P(E) \leq g(Q(E), D(P \| Q))$. Then $g(Q(E), D(P \| Q)) \geq f_{Q(E)}^{-1}(D(P \| Q))$ if $D(P \| Q) \leq \log \frac{1}{Q(E)}$. This is true since given any tuple $(\mathcal{Z}, P, Q, E)$ such that $D(P \| Q) \leq \log \frac{1}{Q(E)}$, there exists $\left(\mathcal{Z}^{\prime}, P^{\prime}, Q^{\prime}, E^{\prime}\right)$ such that $Q\left(E^{\prime}\right)=Q(E), D(P \| Q)=$ $D\left(P^{\prime} \| Q^{\prime}\right)$, and (1) holds with equality. In particular, choose $\mathcal{Z}^{\prime}=\{0,1\}, E^{\prime}=\{1\}, Q^{\prime} \sim \operatorname{Ber}(Q(E))$, and $P^{\prime} \sim \operatorname{Ber}\left(f_{Q(E)}^{-1}(D(P \| Q))\right)$.

However, there is no closed form for the bound in (1). The following corollary provides an upper bound in closed form:

Corollary 1: Given $q \in(0,1 / 2]$, define $g_{q}(y):=$ $\log ^{2}(2)+(\log (1-q)+y)(-\log (q)-y)$ and $\hat{f}_{q}:$ $[0,-\log (q)) \rightarrow \mathbb{R}_{+}$as follows:

$$
\begin{aligned}
& \hat{f}_{q}(y)= \\
& \frac{2 \log ^{2}(2)+(\log (1-q)+y) \log \frac{(1-q)}{q}+(\log 4) \sqrt{g_{q}(y)}}{\log ^{2}((1-q) / q)+\log ^{2}(2)} .
\end{aligned}
$$

Then, $\hat{f}_{q}(y)$ is concave and non-decreasing in $y$. Moreover, given any event $E$ and pair of distributions $P$ and $Q$ with $D(P \| Q) \leq \log \frac{1}{Q(E)}$,

$$
P(E) \leq \hat{f}_{Q(E)}(D(P \| Q)) .
$$

In particular, given an event $E \subseteq \mathcal{X} \times \mathcal{Y}$ and a joint $P_{X Y}$ satisfying $I(X ; Y) \leq \log \frac{1}{P_{X} P_{Y}(E)}$,

$$
P_{X Y}(E) \leq \hat{f}_{P_{X} P_{Y}(E)}(I(X ; Y)) .
$$

Proof: Since $g_{q}(y)$ is concave in $y$ and the square root is concave and non-decreasing, $\sqrt{g_{q}(y)}$ is concave in $y$; hence $\hat{f}_{q}(y)$ is concave in $y$. To show that it is nondecreasing, consider the derivative (ignoring the positive denominator):

$$
\frac{d \hat{f}_{q}(y)}{d y}=\log \frac{1-q}{q}+\log (4) \frac{-2 y-\log (q(1-q))}{2 \sqrt{g_{q}(y)}} .
$$

For $y \in\left[0,-\frac{1}{2} \log (q(1-q))\right]$, both terms are nonnegative (the first is non-negative since $q \leq 1 / 2$ ). For $y \in\left[-\frac{1}{2} \log (q(1-q)),-\log (q)\right]$, the numerator of the second term is negative and decreasing, and the denominator is positive and decreasing. Hence, it achieves its minimum for $y=-\log (q)$. Since the $\left.\operatorname{minimum} \frac{d \hat{f}_{q}(y)}{d y}\right|_{y=-\log (q)}=0$, we get that $\frac{d \hat{f}_{q}(y)}{d y} \geq 0$ for $y \in[0,-\log (q)]$.

Now, let $p:=P(E)$ and $q:=Q(E)$. Then we can rewrite the inequality $D(p \| q) \leq D(P \| Q)$ as

$$
-\log (1-q)+p \log \left(\frac{1-q}{q}\right)-h(p) \leq D(P \| Q),
$$

where $h($.$) is the binary entropy function (in nats).$ Upper-bounding $h(p) \leq(\log 4) \sqrt{p(1-p)}$, we get

$-\log (1-q)+p \log \frac{1-q}{q}-(\log 4) \sqrt{p(1-p)} \leq D(P \| Q)$.

For ease of notation, let $y:=D(P \| Q)$ and $\tilde{g}(p)$ be the left-hand side. Then,

$$
\frac{d \tilde{g}}{d p}=\log \left(\frac{1-q}{q}\right)-(\log 4) \frac{1-2 p}{\sqrt{p(1-p)}} .
$$

Hence, there exists $p_{0}$ such that $\tilde{g}$ is decreasing on $\left[0, p_{0}\right]$ and increasing on $\left[p_{0}, 1\right]$. Therefore, $\tilde{g}(p)=y$ admits at most two solutions, say $p_{1}<p_{2}$, and $\tilde{g}(p) \leq y \Rightarrow p \leq$ $p_{2}$. It remains to solve

$$
p \log \frac{1-q}{q}-\log (1-q)-(\log 4) \sqrt{p(1-p)}=y .
$$

Let $q_{1}=\log \frac{1-q}{q}$, and $q_{2}=\log (1-q)$. We get

$$
\begin{aligned}
\left(p q_{1}-q_{2}-y\right)^{2} & =p(1-p) \log ^{2}(4), \Longleftrightarrow \\
p^{2}\left(q_{1}^{2}+\log ^{2}(4)\right) & -2 p\left(2 \log ^{2}(2)+q_{1}\left(q_{2}+y\right)\right) \\
& +\left(q_{2}+y\right)^{2}=0 .
\end{aligned}
$$

The discriminant of (9) is given by

$$
\begin{aligned}
& \frac{\Delta}{4}=\left(2 \log ^{2}(2)+q_{1}\left(q_{2}+y\right)\right)^{2}-\left(q_{1}^{2}+\log ^{2}(4)\right)\left(q_{2}+y\right)^{2} \\
& =\left(q_{2}+y\right)\left(4 q_{1} \log ^{2}(2)-\left(\log ^{2}(4)\right)\left(q_{2}+y\right)\right)+4 \log ^{4}(2) \\
& =\left(4 \log ^{2}(2)\right)\left(\log ^{2}(2)+\left(q_{2}+y\right)\left(q_{1}-q_{2}-y\right)\right) \geq 0,
\end{aligned}
$$

where the inequality follows from the fact that $q_{1}-q_{2}-$ $y=-\log (q)-y \geq 0$. Hence, the larger root of (9) is given by $\hat{f}_{q}(p)$, as desired.

1) Comparison with existing bounds: It has been shown [12] [14, Lemma 3.11] [13, Lemma 9] that

$$
P(E) \leq \frac{D(P \| Q)+\log (2)}{\log (1 / Q(E))}
$$

The bound in Corollary 1 can be arbitrarily smaller than (10). That is, let $\tilde{f}_{Q(E)}(D(P \| Q))$ be the right-hand side of (10) and consider the calculation shown at the top of the next page. 


$$
\begin{aligned}
& \lim _{q \rightarrow 0} \lim _{D(P \| Q) \rightarrow 0} \frac{\hat{f}_{q}(D(P \| Q))}{\tilde{f}_{q}(D(P \| Q))} \\
& \stackrel{(a)}{=} \lim _{q \rightarrow 0} \frac{\left(2 \log ^{2}(2)+q_{1} q_{2}+(2 \log 2) \sqrt{\log ^{2}(2)-q_{2} \log (q)}\right) \log (1 / q)}{\left(q_{1}^{2}+\log ^{2}(2)\right) \log (2)} \\
& =\lim _{q \rightarrow 0} \frac{\left(2 \log ^{2}(2)+\log ^{2}(1-q)-\log (q) \log (1-q)+(2 \log 2) \sqrt{\log ^{2}(2)-\log (1-q) \log (q)}\right) \log (1 / q)}{\left(\log ^{2}(1-q)+\log ^{2}(q)-2 \log (q) \log (1-q)+\log ^{2}(2)\right) \log (2)} \\
& \stackrel{(b)}{=} \lim _{q \rightarrow 0} \frac{4 \log ^{2}(2) \log (1 / q)}{\left(\log ^{2}(q)+\log ^{2}(2)\right) \log (2)} \\
& =0,
\end{aligned}
$$

where in (a) $q_{1}=\log \frac{1-q}{q}$ and $q_{2}=\log \left(1-q\right.$ ), and (b) follows from the fact that $\lim _{q \rightarrow 0} \log (q) \log (1-q)=0$.

Moreover, one can derive a family of bounds in the form of (10) using the Donsker-Varadhan characterization of the KL divergence. In particular,

$$
D(P \| Q)=\sup _{f: \mathcal{Z} \rightarrow \mathbb{R}, \mathbf{E}_{Q}\left[e^{f}\right]<+\infty}\left\{\mathbf{E}_{P}[f]-\log \mathbf{E}_{Q}\left[e^{f}\right]\right\} .
$$

Now, let $f=\beta \mathbb{I}\{z \in E\}$ for some $\beta>0$, where $\mathbb{I}\{\}$ is the indicator function. After rearranging terms, we get

$$
P(E) \leq \frac{D(P \| Q)+\log \left(1+\left(e^{\beta}-1\right) Q(E)\right)}{\beta} .
$$

Choosing $\beta=\log (1 / Q(E))$, we slightly improve (10) by replacing $\log (2)$ with $\log (2-Q(E))$. In fact, we can solve the infimum over $\beta>0$ of the right-hand side of (12). In particular, by [18, Lemma 2.4], the infimum is given by $\ell^{\star-1}(D(P \| Q))$, where $\ell^{\star}$ is the convex conjugate of ${ }^{1} \ell(\beta)=\log \left(1+\left(e^{\beta}-1\right) Q(E)\right)$, and $\ell^{\star-1}(y)=\inf \left\{t: \ell^{\star}(t)>y\right\}$. It turns out that $\ell^{\star}: \mathbb{R}_{+} \rightarrow \mathbb{R}_{+}$is given by

$$
\ell^{\star}(t)= \begin{cases}0, & 0 \leq t<Q(E), \\ D(t|| Q(E)), & Q(E) \leq t \leq 1, \\ +\infty, & t>1 .\end{cases}
$$

Now, $P(E) \leq \inf \left\{t: \ell^{\star}(t)>D(P \| Q)\right\}$. Hence, for $D(P \| Q)=0, P(E) \leq \inf (Q(E),+\infty)=Q(E)$. By noting that $\ell^{\star}(1)=\log (1 / Q(E))$, we get for any $D(P \| Q)>\log (1 / Q(E)), P(E) \leq \inf (1,+\infty)=$ 1. Finally, for $D(P \| Q) \in(0, \log (1 / Q(E))]$, we get $P(E) \leq\{t \in[Q(E), 1]: D(t|| Q(E))>$ $D(P \| Q)\}$, which is equal to $t^{\star} \in[Q(E), 1]$ satisfying

\footnotetext{
${ }^{1}$ Lemma 2.4 of [18] assumes $\ell^{\prime \prime}(0)=0$, but the proof goes as is for $\ell^{\prime \prime}(0) \geq 0$, which is the case here.
}

$D\left(t^{\star} \| Q(E)\right)=D(P \| Q)$. That is, the bound derived from (13) exactly recovers Proposition 1.

Furthermore, we could compare with the mutual information bound of Russo and Zou [8], and Xu and Raginsky [9]. In particular, by considering $f=$ $\beta(\mathbb{I}\{z \in E\}-Q(E))$ for $\beta \in \mathbb{R}$ in (11), we get

$$
\begin{aligned}
D(P \| Q) & \geq \beta(P(E)-Q(E))-\log \mathbf{E}_{Q}\left[e^{(\mathbb{I}\{Z \in E\}-Q(E))}\right] \\
& \geq \beta(P(E)-Q(E))-\beta^{2} / 8,
\end{aligned}
$$

where the second inequality follows from the fact that $(\operatorname{Ber}(q)-q)$ is $\frac{1}{4}$-subgaussian (which is true for any random variable whose support has length 1 ). Since the above inequality holds for any $\beta \in \mathbb{R}$, we get

$$
P(E) \leq Q(E)+\sqrt{\frac{D(P \| Q)}{2}} .
$$

Given the form of the 3 bounds, one might expect that (14) outperforms the other two for large values of $D(P \| Q)$. This is in fact not true because the range of interest for the right-hand sides is restricted to $[0,1]$. For instance, for small $Q(E)$ and $D(P \| Q)=$ $-\log (Q(E)) / 2$, the bound in (14) is trivial $(>1)$, and the other two bounds are strictly less than 1 .

\section{B. Lautum Information Bounds}

By considering the data processing inequality $D(q \| p) \leq D(Q \| P)$, we can bound $p$ in terms of $q$ and $D(Q \| P)$.

Theorem 2: Given any event $E$ and a pair of distributions $P$ and $Q$, if $P(E) \leq 1 / 2$, then

$$
P(E) \leq 1-e^{-h(Q(E))-D(Q \| P)} .
$$


In particular, given an event $E \subseteq \mathcal{X} \times \mathcal{Y}$ and a joint distribution $P_{X Y}$ with $P_{X Y}(E) \leq 1 / 2$,

$$
P_{X Y}(E) \leq 1-e^{-h\left(P_{X} P_{Y}(E)\right)-L(X ; Y)},
$$

where $L(X ; Y):=D\left(P_{X} P_{Y} \| P_{X Y}\right)$ is the lautum information [15].

Proof: Set $p=P(E)$ and $q=Q(E)$. As in (6), we can rewrite $D(q \| p) \leq D(Q \| P)$ as

$$
q \log \left(\frac{1-p}{p}\right)-\log (1-p)-h(q) \leq D(Q \| P) .
$$

Since $p \leq 1 / 2$ (by assumption), we can drop the first term of the left-hand side. Rearranging the inequality then yields Theorem 2 .

Moreover, we can derive a family of bounds similar to (12) by considering the Donsker-Varadhan representation of $D(Q \| P)$ :

$$
D(Q \| P)=\sup _{f: \mathcal{Z} \rightarrow \mathbb{R}, \mathbf{E}_{P}\left[e^{f}\right]<+\infty}\left\{\mathbf{E}_{Q}[f]-\log \mathbf{E}_{P}\left[e^{f}\right]\right\} .
$$

Now, let $f=-\beta \mathbb{I}\{z \in E\}$ for some $\beta>0$. Then after rearranging terms, we get for any $\beta>0$,

$$
P(E) \leq \frac{1-e^{-D(Q \| P)-\beta Q(E)}}{1-e^{-\beta}} .
$$

\section{MAXIMAL LEAKAGE BOUND}

The bounds presented so far in (4) and (15) do not take into account the specific relation of $P_{X Y}$ and $P_{X} P_{Y}$ as a joint distribution and its marginal. Indeed, they are applications of a more general bound that can be applied to an arbitrary pair of distributions (Corollary 1 and Theorem 2). The following bound does not fall under this category, i.e., it only applies to pairs of distributions forming a joint and marginal.

Theorem 3: Given $\alpha \in[0,1]$, finite alphabets $\mathcal{X}$ and $\mathcal{Y}$, a joint distribution $P_{X Y}$ and an event $E \subseteq \mathcal{X} \times \mathcal{Y}$ such that for all $y \in \mathcal{Y}, P_{X}\left(E_{y}\right) \leq \alpha$ where $E_{y}:=\{x:$ $(x, y) \in E\}$, then

$$
P_{X Y}(E) \leq \alpha \exp \{\mathcal{L}(X \rightarrow Y)\},
$$

where $\mathcal{L}(X \rightarrow Y)=\log \sum_{y \in \mathcal{Y}} \max _{x: P_{X}(x)>0} P_{Y \mid X}(y \mid x)$ is the maximal leakage.

Remark 2: The bound holds more generally but we restrict our attention to finite alphabets to make the presentation of the proof simple.

Remark 3: A similar inequality appeared (without proof) in [19].

Maximal leakage has recently appeared in the information theory literature [17] as an operational measure of information leakage:
Definition 1: Given a joint distribution $P_{X Y}$ on finite alphabets $\mathcal{X}$ and $\mathcal{Y}$, the maximal leakage from $X$ to $Y$ is defined as

$$
\mathcal{L}(X \rightarrow Y)=\sup _{U-X-Y-\hat{U}} \log \frac{\operatorname{Pr}(U=\hat{U})}{\max _{u \in \mathcal{U}} P_{U}(u)},
$$

where $U$ and $\hat{U}$ take values in the same finite, but arbitrary, alphabet.

That is, given $X$ and $Y, \mathcal{L}(X \rightarrow Y)$ is given by (the logarithm of) the multiplicative increase of the probability of guessing any (possibly randomized) function of $X$ by observing $Y$ (as compared with no observations). Hence, as a leakage metric, one can view maximal leakage as controlling the degree of dependence between the input and the output.

Proof of Theorem 3: Fix $y \in \mathcal{Y}$ satisfying $P_{Y}(y)>0$, and consider the pair of distributions $P_{X \mid Y=y}$ and $P_{X}$ :

$$
\begin{aligned}
\exp \left\{D_{\infty}\left(P_{X \mid Y=y}|| P_{X}\right)\right\} & =\sup _{A \subseteq \mathcal{X}} \frac{P_{X \mid Y=y}(A)}{P_{X}(A)} \\
& =\max _{x: P_{X \mid Y}(x \mid y)>0} \frac{P_{X \mid Y}(x \mid y)}{P_{X}(x)} .
\end{aligned}
$$

where the equalities follow from [20, Theorem 6]. Hence,

$$
\begin{aligned}
P_{X \mid Y=y}\left(E_{y}\right) & \leq \alpha \max _{x: P_{X \mid Y}(x \mid y)>0} \frac{P_{X \mid Y}(x \mid y)}{P_{X}(x)} \\
& =\alpha \max _{x: P_{X \mid Y}(x \mid y)>0} \frac{P_{Y \mid X}(y \mid x)}{P_{Y}(y)} .
\end{aligned}
$$

Now,

$$
\begin{aligned}
P_{X Y}(E) & =\mathbf{E}_{Y}\left[P_{X \mid Y=y}\left(E_{y}\right)\right] \\
& \leq \alpha \sum_{y: P_{Y}(y)>0} \max _{x: P_{X \mid Y}(x \mid y)>0} P_{Y \mid X}(y \mid x) \\
& \stackrel{(a)}{=} \alpha \sum_{y: P_{Y}(y)>0} \max _{x: P_{X}(x)>0} P_{Y \mid X}(y \mid x) \\
& =\alpha \sum_{y \in \mathcal{Y}} \max _{x: P_{X}(x)>0} P_{Y \mid X}(y \mid x)
\end{aligned}
$$

where (a) follows from the following (readily verifiable) facts:

$$
\begin{aligned}
& P_{Y}(y)>0 \text { and } P_{X \mid Y}(x \mid y)>0 \Rightarrow P_{X}(x)>0, \\
& P_{Y}(y)>0 \text { and } P_{X \mid Y}(x \mid y)=0 \Rightarrow P_{Y \mid X}(y \mid x)=0 .
\end{aligned}
$$

The bound of Theorem 3 outperforms the bound in (10) if and only if

$$
\frac{e^{\mathcal{L}(X \rightarrow Y)}}{I(X ; Y)+\log 2} \leq \frac{1}{\alpha \log (1 / \alpha)} .
$$


In applications of interest, the input consists of $n$ i.i.d samples, and $\alpha$ is exponentially small. The above inequality thus holds in certain cases of interest.

One advantage of the bound of Theorem 3 is that it depends on a partial description of $P_{Y \mid X}$ only. By contrast, maximizing the mutual information bounds over $P_{X}$ would not yield a closed-form solution. Hence, the above bound is simpler to analyze than the mutual information bounds. Moreover, for fixed $P_{X}$, the bound is convex in $P_{Y \mid X}$. In the next subsection, we present a bound with similar properties.

\section{IV. $J_{\infty}$-BOUND}

Theorem 4: Given $\alpha \in[0,1 / 2]$, finite alphabets $\mathcal{X}$ and $\mathcal{Y}$, a joint distribution $P_{X Y}$ and an event $E \subseteq \mathcal{X} \times \mathcal{Y}$ such that for all $y \in \mathcal{Y}, P_{X}\left(E_{y}\right) \leq \alpha$ where $E_{y}:=\{x$ : $(x, y) \in E\}$, then

$$
P_{X Y}(E) \leq \alpha\left(2(1-\alpha) J_{\infty}(X ; Y)+1\right),
$$

where $\quad J_{\infty}(X ; Y)$ $\frac{1}{2} \sum_{y \in \mathcal{Y}}\left(\max _{x} P_{Y \mid X}(y \mid x)-\min _{x} P_{Y \mid X}(y \mid x)\right)$ [11].

Proof: The theorem follows from Theorem 1 and Corollary 1 of [11]. In particular, following the same proof steps as in [11], one can show that for any function $^{2} f: \mathcal{X} \times \mathcal{Y} \rightarrow \mathbb{R}$

$$
\begin{aligned}
& \left|\mathbf{E}_{P_{X Y}}[f(X, Y)]-\mathbf{E}_{P_{X} P_{Y}}[f(X, Y)]\right| \leq \\
& \left(\max _{y} \mathbf{E}_{P_{X}}\left[\left|f(X, y)-\mu_{y}\right|\right]\right) J_{\infty}(X ; Y),
\end{aligned}
$$

where $\mu_{y}:=\mathbf{E}_{P_{X}}[f(X, y)]$. Now, set $f(x, y)=$ $\mathbb{I}\{(x, y) \in E\}$. Then, $\mathbf{E}_{P_{X Y}}[f(X, Y)]=P_{X Y}(E)$, $\mathbf{E}_{P_{X} P_{Y}}[f(X, Y)]=P_{X} P_{Y}(E) \leq \alpha$, and $\mathbf{E}_{P_{X}}[f(X, y)]=P_{X}\left(E_{y}\right)$. Moreover,

$$
\begin{aligned}
\mathbf{E}_{P_{X}}\left[\left|f(X, y)-P_{X}\left(E_{y}\right)\right|\right] & =2 P_{X}\left(E_{y}\right)\left(1-P_{X}\left(E_{y}\right)\right) \\
& \leq \alpha(1-\alpha),
\end{aligned}
$$

where the last inequality follows from the assumption that $P_{X}\left(E_{y}\right) \leq \alpha \leq \frac{1}{2}$. Then, it follows from (21) that

$$
P_{X Y}(E)-P_{X} P_{Y}(E) \leq 2 \alpha(1-\alpha) J_{\infty}(X ; Y) .
$$

The theorem follows by noting that $P_{X} P_{Y}(E) \leq \alpha$.

\section{ACKNOWLEDGMENT}

This work was supported in part by the Swiss National Science Foundation under Grant 169294.

${ }^{2}$ In [11], the authors consider $X=\left(X_{1}, \cdots, \mathcal{X}_{n}\right), \mathcal{Y}=$ $\{1,2, \cdots, n\}$, and $f(X, Y)=X_{Y}$. Nevertheless, the proof of (21) remains the same.

\section{REFERENCES}

[1] J. P. Ioannidis, "Why most published research findings are false," PLoS medicine, vol. 2, no. 8, p. e124, 2005.

[2] J. P. Simmons, L. D. Nelson, and U. Simonsohn, "False-positive psychology: Undisclosed flexibility in data collection and analysis allows presenting anything as significant," Psychological science, vol. 22, no. 11, pp. 1359-1366, 2011.

[3] M. Hardt and J. Ullman, "Preventing false discovery in interactive data analysis is hard," in Foundations of Computer Science (FOCS), 2014 IEEE 55th Annual Symposium on. IEEE, 2014, pp. 454-463.

[4] S. Shalev-Shwartz, O. Shamir, N. Srebro, and K. Sridharan, "Learnability, stability and uniform convergence," Journal of Machine Learning Research, vol. 11, no. Oct, pp. 2635-2670, 2010.

[5] C. Dwork, V. Feldman, M. Hardt, T. Pitassi, O. Reingold, and A. L. Roth, "Preserving statistical validity in adaptive data analysis," in Proceedings of the forty-seventh annual ACM symposium on Theory of computing. ACM, 2015, pp. 117-126.

[6] C. Dwork, V. Feldman, M. Hardt, T. Pitassi, O. Reingold, and A. Roth, "Generalization in adaptive data analysis and holdout reuse," CoRR, vol. abs/1506.02629, 2015. [Online]. Available: http://arxiv.org/abs/1506.02629

[7] R. Bassily, K. Nissim, A. Smith, T. Steinke, U. Stemmer, and J. Ullman, "Algorithmic Stability for Adaptive Data Analysis," ArXiv e-prints, Nov. 2015.

[8] D. Russo and J. Zou, "Controlling bias in adaptive data analysis using information theory," in Artificial Intelligence and Statistics, 2016, pp. 1232-1240.

[9] A. Xu and M. Raginsky, "Information-theoretic analysis of generalization capability of learning algorithms," arXiv preprint arXiv:1705.07809, 2017.

[10] J. Jiao, Y. Han, and T. Weissman, "Dependence measures bounding the exploration bias for general measurements," in Proc. IEEE Int. Symp. Inf. Theory (ISIT). IEEE, June 2017, pp. 1475-1479.

[11] I. Issa and M. Gastpar, "Computable bounds on the exploration bias," in Proc. IEEE Int. Symp. Inf. Theory (ISIT), June 2018, pp. $576-580$.

[12] E. A. Arutjunjan, "Bounds for the Exponent of the Probability of Error for a Semicontinuous Memoryless Channel," Probl. Peredachi Inf., vol. 4, no. 4, pp. 37-48, 1968.

[13] R. Bassily, S. Moran, I. Nachum, J. Shafer, and A. Yehudayoff, "Learners that use little information," in Proceedings of Algorithmic Learning Theory, ser. Proceedings of Machine Learning Research, F. Janoos, M. Mohri, and K. Sridharan, Eds., vol. 83. PMLR, 07-09 Apr 2018, pp. 25-55. [Online]. Available: http://proceedings.mlr.press/v83/bassily18a.html

[14] V. Feldman and T. Steinke, "Calibrating noise to variance in adaptive data analysis," arXiv preprint arXiv:1712.07196, 2017.

[15] D. P. Palomar and S. Verdú, "Lautum information," IEEE transactions on information theory, vol. 54, no. 3, pp. 964-975, 2008.

[16] C. Braun, K. Chatzikokolakis, and C. Palamidessi, "Quantitative notions of leakage for one-try attacks," Electronic Notes in Theoretical Computer Science, vol. 249, pp. 75-91, 2009.

[17] I. Issa, S. Kamath, and A. B. Wagner, "An operational measure of information leakage," in Proc. of 50th Ann. Conf. on Information Sciences and Systems (CISS), Mar. 2016.

[18] S. Boucheron, G. Lugosi, and P. Massart, Concentration inequalities: A nonasymptotic theory of independence. Oxford university press, 2013.

[19] A. D. Smith, "Information, privacy and stability in adaptive data analysis," CoRR, vol. abs/1706.00820, 2017.

[20] T. van Erven and P. Harremos, "Rényi divergence and KullbackLeibler divergence," IEEE Trans. Inf. Theory, vol. 60, no. 7, pp. 3797-3820, July 2014. 